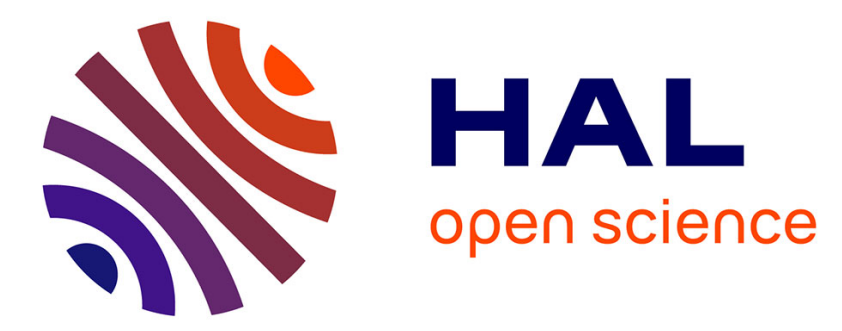

\title{
A Group Evacuation Method for Smart Buildings
}

Qasim Khalid, Alberto Fernández, Marin Lujak, Arnaud Doniec

\section{To cite this version:}

Qasim Khalid, Alberto Fernández, Marin Lujak, Arnaud Doniec. A Group Evacuation Method for Smart Buildings. 10th Doctoral Conference on Computing, Electrical and Industrial Systems (DoCEIS), May 2019, Costa de Caparica, Portugal. pp.206-213, 10.1007/978-3-030-17771-3_17 . hal02295233

\section{HAL Id: hal-02295233 \\ https://hal.inria.fr/hal-02295233}

Submitted on 24 Sep 2019

HAL is a multi-disciplinary open access archive for the deposit and dissemination of scientific research documents, whether they are published or not. The documents may come from teaching and research institutions in France or abroad, or from public or private research centers.
L'archive ouverte pluridisciplinaire HAL, est destinée au dépôt et à la diffusion de documents scientifiques de niveau recherche, publiés ou non, émanant des établissements d'enseignement et de recherche français ou étrangers, des laboratoires publics ou privés. 


\title{
A Group Evacuation Method for Smart Buildings
}

\author{
Qasim Khalid ${ }^{1}$, Alberto Fernández ${ }^{1}$, Marin Lujak², and Arnaud Doniec ${ }^{2}$ \\ ${ }^{1}$ CETINIA, Universidad Rey Juan Carlos, Madrid, Spain, \\ \{qasim.khalid, alberto.fernandez\}@urjc.es \\ ${ }^{2}$ IMT Lille Douai, Univ. Lille, Unité de Recherche Informatique Automatique, F-59000 \\ Lille, France, \{marin.lujak, arnaud.doniec\}@imt-lille-douai.fr
}

\begin{abstract}
Mass evacuation of people in buildings during emergencies is still a burning question regarding safety and reliability. Due to lack of information, crowd prefers to evacuate in the form of random groups. This random grouping increases the possibility of panic among evacuees due to human behavioral factors like herding and stampeding. As a result, congestion may occur resulting in unnecessary casualties. For this purpose, we propose a multi-agent evacuation architecture, that not only collects all the information from evacuees and events occurring in the building on a real-time basis but also provides the evacuation routes to evacuees. In this paper, we discuss a group formation module of our proposed architecture and use an example as a test case to check the functionality of our proposed group formation approach.
\end{abstract}

Keywords: Multi-agent system, evacuation, semantic technology, situation awareness, smart buildings

\section{Introduction}

We would like to draw your attention to the fact that it is not possible to modify a paper in any way, once it has been published. This applies to both the printed book and the online version of the publication. Every detail, including the order of the names of the authors, should be checked before the paper is sent to the Volume Editors.

Emergency evacuation is performed in case of fire, terrorist threat, earthquake or any other hazardous situation in buildings. The ultimate goal of evacuation for all the occupants is to move out of a specific area or a building safely and as quick as possible. In general, a static route provided in an evacuation plan is followed, which is developed during the erection stage of a building. However, static evacuation plans lack coordination between evacuees and administration as it does not consider contingencies that may occur during evacuation. Due to the absence of any coordination during the whole evacuation process, several problems may occur, e.g., congestion along evacuation routes or at exit points or locations that are frequently used, and as a result, casualties may occur. Among important factors associated with an emergency evacuation are evacuees' behavior, their mobility characteristics, and their coordination in following the evacuation route related to a type of emergency 
and relevant evacuation strategy. Vermuyten et al. have conducted a literature survey in which they discussed various evacuation strategies and crowd behavior, crowd flow, optimized paths, bottlenecks, etc. (see, e.g., [1]). These strategies have somehow improved the evacuation process, but most of the studies are static. Due to this reason, real-time status of buildings and evacuees is not known in case of an emergency and during the evacuation process. Therefore, there is still a need for an intelligent system that could detect hazardous situations and handle evacuation problem on a real-time basis.

To this end, we propose a real-time multi-agent architecture for mass evacuation in smart buildings. The novel concept for this study is that the information of events and evacuees is processed on a microscopic level and the evacuation is done in the form of groups on a macroscopic level. These groups are created on the basis of momentary location, possible family ties with other evacuees, and mobility characteristics of each evacuee. Later, groups evacuate the building under the supervision of their assigned group leaders. These group leaders use hand-held devices that provide all the information needed for the evacuation process. At this point, our system is designed for the office spaces and the places where people are slightly aware of topology of the buildings. Due to the scope of this paper, we briefly discuss our proposed architecture and its functioning. Also, we specifically focus on the group formation module of the proposed architecture.

The rest of the paper is organized as follows. Section 2 states the contribution of this research to service systems. Related works in the area of crowd evacuation and smart buildings with their limitations are discussed in Section 3. The structure of the proposed architecture is explained in Section 4. The group formation methodology is discussed in Section 5 and we give a functioning example in Section 6. In the end, we conclude the paper with further research directions in Section 7.

\section{Relationship to Industrial and Service Systems}

In a service system, both technology and manpower work together to fulfill the requirements of its user(s). As service systems maximize the output as well as ensure the safety and reliability of the system, therefore its applications are found everywhere such as in the sectors of engineering, banking, supermarkets, and even on internet.

The main objective of service systems is to serve its customers and provide them what they want in minimum time. Customer satisfaction is also an important factor in this type of systems. A service system must be safe and reliable enough so that its users can use it without any risk or danger. When it comes to the cyber-physical world where machines perform most of the work, while humans are involved mostly as users, then the question of user satisfaction and safety is of special importance.

In this study, we propose an intelligent architecture for evacuation of buildings. The purpose of this system is to minimize the involvement of a specialized personnel for evacuation management during any emergency situation, while providing decision support for evacuation of a multitude of people according to their evacuation requirements and preferences, such as mobility characteristics and mutual family ties. 
Since the lives of people are dependent on it, the reliability of such a system is of utmost priority. In this respect, the proposed decision support architecture for emergency evacuation may be seen as a service system whose aim is to minimize the evacuation related risks considering the mobility requirements of its users.

\section{Related Work}

Multiple approaches have been proposed for the emergency evacuation of buildings (see, e.g., [2-5]). Vermuyten et al. performed a literature survey in which they have classified the research topics according to the phases and requirements of the whole evacuation system, e.g., pedestrian dynamics, evacuation design, optimization of evacuation routes, crowd management, model movement, and crowd flow (see, e.g., [1]).

There are generally two kinds of crowd evacuation models: macroscopic and microscopic one. Huges explained the significance and the applicability of both approaches for handling the crowd in [6]. The macroscopic model is used at a crowd level to implement the rules over a whole crowd whereas the microscopic model is used to model every individual participating in the evacuation process. Here, some authors have proposed a hybrid approach to use both strategies as they complement each other (see, e.g., [7]).

On the other hand, Liu et al. explained that the evacuation process is not only individual action, but it is a group activity where the efficiency of the whole process is dependent on everyone involved. Hence, coordination and cooperation of evacuees is needed to make that evacuation process efficient (see, e.g., $[8,9])$. In this manner, researchers have proposed some interesting strategies to observe the behavior of groups during an evacuation. For example, [10] developed a genetic algorithm with a combination of a cellular automata model to evacuate groups of people. In this model, each group is assigned to a specific exit which evacuates within a specific time because the next group has to evacuate afterwards. The best evacuation plan is found after finding the route having minimum evacuation time.

On the other hand, when the crowd evacuates in the form of groups, synchronization and coordination issues arise. In case of any wrong decision by the group leader, even worse disaster could happen as the time is a most critical thing during that process (see, e.g., [11]). However, it is also found that the evacuation time increases because of the presence of groups in the crowd (see, e.g., [12]).

A hybrid evacuation strategy that could assist the evacuees in the formation of groups as well as in choosing an optimal route for them is still an open challenge that we treat in this paper.

\section{Evacuation Architecture}

In this section, we explain the architecture of our proposed system for evacuation. The system consists of three agents namely, Situation Awareness Agent, Route Optimization Agent, and Group Management Agent. Situation Awareness Agent 
handles and processes the information of events coming from sensors on a real-time basis. Various sensors are installed that detect fire, smoke, terrorist attack or threat, quarrel or any other risk that could lead to an emergency evacuation (see, e.g., [1315]). This agent also communicates with other agents to assign an alternate safe route to evacuees in case of any blockage or congestion in the existing provided path. Route Optimization Agent chooses the optimal exit path for each group. For this purpose, the building is represented as a graph and divided into different sections called nodes, and the paths that connect these sections called edges or links. Later, the safest and fastest route is calculated with the help of the live information received from the sensors. Group Management Agent creates groups which are supposed to evacuate together under the supervision of (human) group leaders. Groups are created using evacuees' live locations, family ties and physical characteristics whereas group leaders are trained occupants of a building chosen beforehand on a voluntary basis. This agent provides the formation of groups with their relevant collection points and the allocation of groups' leaders. The block diagram of the architecture is shown in Fig. 1 .

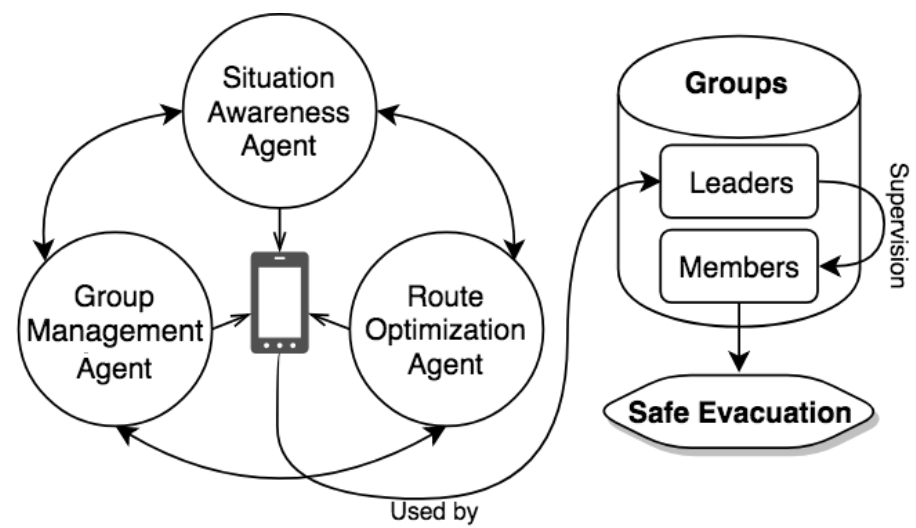

Fig. 1. Evacuation Architecture.

Personal information of evacuees related to evacuation is crucial for wellfunctioning of the proposed system and should be obtained beforehand. As this information is static, it is stored in the database with the help of an ontology. Upon entering the details into the system, a special RFID or a class of Bluetooth low energy (BLE) device (e.g., iBeacon) tag is provided to each person which they have to wear all the time during the evacuation so that all the movements and locations of people are monitored.

As it is discussed in [16], the evacuation process is a multi-phase procedure. Therefore, we divide the whole evacuation process into two phases: notifying phase and evacuation phase. Firstly, during the notification phase, we use an audio or a text message or an email to inform every evacuee about his/her assigned group collection point. Also, this information (based on the momentary location) is shown on public screen displays. During the evacuating phase, real-time location of every occupant is 
processed individually, and groups are updated on the basis of this information. Later, the groups gather at their relevant collection points, and then group leaders start the evacuation process under their supervision. Note that the group leaders use smart devices with an app where all information of group members are shown along with their calculated evacuation route plans.

\section{Group Formation}

In this section, we focus on the group formation module of group management agent of the proposed architecture.

In general, groups are formed by combining the static and dynamic information of evacuees. Static information includes information of mobility characteristics and family ties with other evacuees. Dynamic information consists of live locations of the people which are continuously detected by sensors. Every person in the building has a RFID or iBeacon tag which is provided before entering into the building or during the registration process. These tags have a unique ID by which the data of every person can be accessed.

If any hazard or risk is detected and evacuation is necessary, evacuees are provided by a location (known as collection points) where they have to gather and wait for next instructions from their group leaders. These collection points are assigned individually in line with the mobility characteristics of each evacuee. In this regard, a procedure for group creation is given in the following. This procedure also provides collection points to evacuees individually.

The procedure requires the following information: i) an updated momentary location and mobility characteristics and family ties for each evacuee, ii) location and capacity of each collection point, iii) available evacuation routes with their free-flow capacities from each one of the collection points, iv) maximum evacuation time given by the evacuation authority.

The procedure runs in the following steps that assign each evacuee to its collection point considering the capacity of the collection points and their evacuation routes.

Step 1: Calculate the shortest evacuation time of each person from his/her momentary location to each collection point;

Step 2: Assign each evacuee whose evacuation time is larger than the maximum evacuation time to the closest collection point with the shortest evacuation time;

Step 3: Assign each mobility impaired person to his/her evacuation leader and a collection point with sufficient capacity and the shortest evacuation time;

Step 4: Assign evacuees with family ties (members of the same family) to the same collection point. In case they are not located in the same area, choose a collection point that is the closest to the same distance from all of them;

Step 5: Assign the rest of the evacuees to their nearest collection points based on the available capacity. 


\section{Case Study}

In this section, we use an example as a test case to check the functionality of our proposed algorithm for group formation of people and their allocation to their feasible collection points. For this purpose, the output of the algorithm is shown in Fig 2.

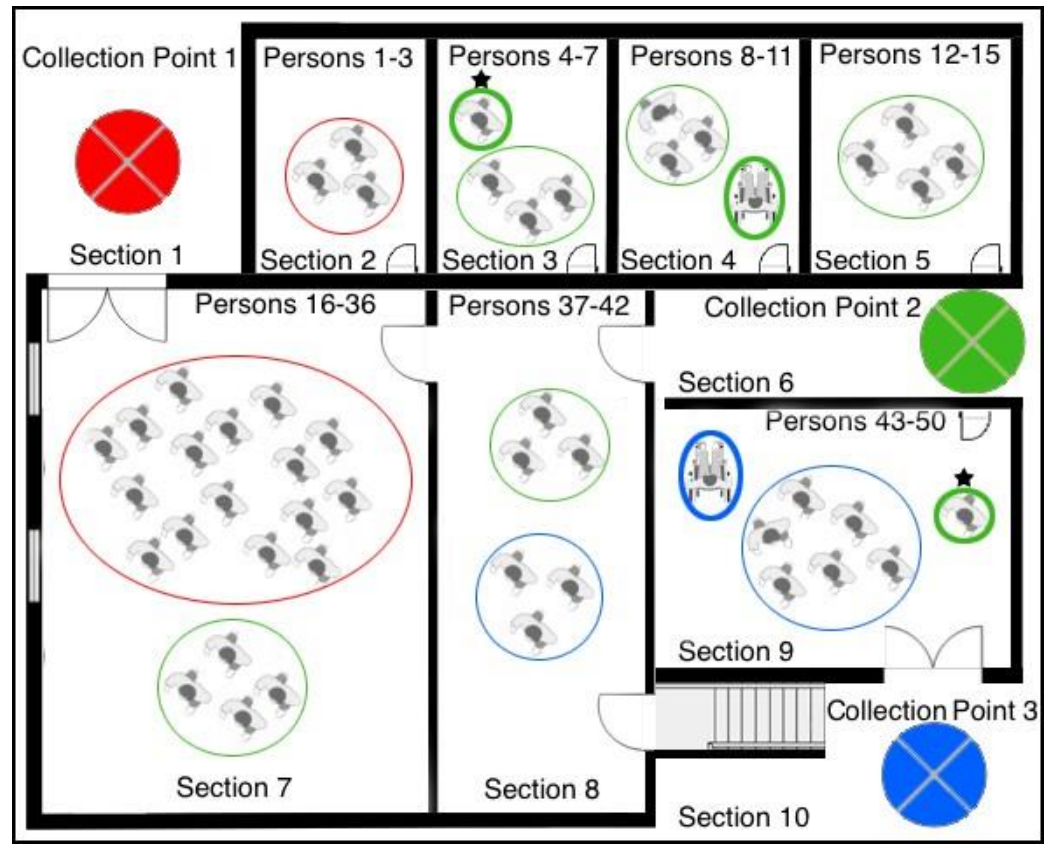

Fig. 2. Final grouping with respect to their feasible collection points.

Given is a layout of a floor in which people are located in possibly different sections. These sections can be rooms and/or corridors, and all sections are connected to every collection point. For simplicity, we assume that the geo-positioning of evacuees is flawless.

In our example in Fig. 2, there are three collection points and 50 persons of which two are mobility impaired (P8, P43) and other two have family ties (P5, P45). We assume that all people are located in the areas that are within the maximum evacuation time from safe exits. Therefore, the priority will be given to mobility impaired people and people with family ties. As a result, P8 and P43, who are mobility impaired are assigned to their nearest collection points, CP2 and CP3 respectively. Also, persons with family ties, P5 and P45, are assigned to the same group/collection point, $\mathrm{CP} 2$, because collection point $\mathrm{CP} 2$ is the nearest one to both of them. The rest of the persons are allocated to their nearest collection point based on the available capacity. Once the capacity of a collection point is reached, remaining persons are allocated to the nearest collection point with available capacity. 
In Fig. 2, collection points are colored in green, red, and blue. People are grouped in groups based on their assigned collection points (red, green, and blue) so that they have to gather at their allocated collection point. People who are mobility impaired have a bigger ring around them to represent their priority. Similarly, people who have family ties also have a bigger ring around them as well as a star over them to distinguish them from able-bodied and mobility impaired people.

The output of the proposed procedure will be in the form of a couple person's ID, collection point of that person (assigned group).

Following are the sets of persons who are allocated to a specific collection point on the basis of knowledge acquired by them. Red, green, and blue sets are formed according to the capacity of collection point 1 , collection point 2 and collection point 3 respectively.

Red set $=\{P 1, P 2, P 3, P 16, P 17, P 18, P 19, P 20, P 21, P 22, P 23, P 24, P 25, P 26$, $P 27, P 28, P 29, P 30, P 31, P 32\}$

Green set $=\{P 5, P 45, P 8, P 4, P 6, P 7, P 9, P 10, P 11, P 12, P 13, P 14, P 15, P 33$, $P 34, P 35, P 36, P 40, P 41, P 42\}$

Blue set $=\{P 45, P 37, P 38, P 39, P 44, P 46, P 47, P 48, P 49, P 50\}$

\section{Conclusion}

In this study, we proposed an evacuation architecture that coordinates the evacuation process from the detection of the hazard or emergency to the evacuation of people in buildings. Our system is based on three different agents that perform their evacuationrelated tasks. In our proposed strategy, evacuation is done in the form of small groups, each one led by a group leader. Each group leader gets live information from an app installed on a smart device specifically designed as an interface to our proposed architecture. In this paper, we discussed the module of group management agent, a module of our proposed architecture together with group formation and allocation of collection points. Also, due to the hierarchy of this solution proposed for buildings, people may have some training for specific scenarios.

As a future work, we plan to develop other agents of our proposed architecture. Also, we will integrate the group formation module, developed in this study, with other parts of the architecture. We also plan to compare the performance of the proposed system with other state-of-the-art evacuation coordination systems.

Acknowledgments. Work partially supported by the Autonomous Region of Madrid (grant "MOSI-AGIL-CM" (S2013/ICE-3019) co-funded by EU Structural Funds FSE and FEDER), project "SURF" (TIN2015-65515-C4-4-R (MINECO/FEDER)) funded by the Spanish Ministry of Economy and Competitiveness, and through the Excellence Research Group GES2ME (Ref. 30VCPIGI05) co- funded by URJCSantander Bank. 


\section{References}

1. Vermuyten, H., Beliën, J., De Boeck, L., Reniers, G. Wauters, T.: A review of optimisation models for pedestrian evacuation and design problems. Safety science, 87:167-178, 2016.

2. Lujak, M., Billhardt, H., Dunkel, J., Fernández, A., Hermoso, R., Ossowski, S.: A distributed architecture for real-time evacuation guidance in large smart buildings. Comput. Sci. Inf. Syst., 14(1):257-282, 2017.

3. Lujak, M and Ossowski, S: Intelligent people flow coordination in smart spaces. In MultiAgent Systems and Agreement Technologies, pages 34-49. Springer, 2015.

4. Wagner, $\mathrm{N}$ and Agrawal, V.: An agent-based simulation system for concert venue crowd evacuation modeling in the presence of a fire disaster. Expert Systems with Applications, 41(6):2807-2815, 2014.

5. Zheng, X., Zhong, T., and Liu, M.: Modeling crowd evacuation of a building based on seven methodologic al approaches. Building and Environment, 44(3):437-445, 2009.

6. Hughes, R.L.: A continuum theory for the flow of pedestrians. Transportation Research Part B: Methodological, 36(6):507-535, 2002.

7. Xiong, M., Tang, S., Zhao, D.: A hybrid model for simulating crowd evacuation. New Generation Computing, 31(3):211-235, 2013.

8. Liu, H.: Context-aware agents in cooperative design environment. International Journal of Computer Applications in Technology, 39(4):187-198, 2010.

9. Liu, H., Sun, Y., Li, Y.: Modeling and path generation approaches for crowd simulation based on computational intelligence. Chinese Journal of Electronics, 21(4):636-641, 2012.

10.Abdelghany, A., Abdelghany, K., Mahmassani, H., Alhalabi, W.: Modeling framework for optimal evacuation of large-scale crowded pedestrian facilities. European Journal of Operational Research, 237(3):1105-1118, 2014.

11.Oxendine, C., Sonwalkar, M., Waters, N.: A multi-objective, multi-criteria approach to improve situational awareness in emergency evacuation routing using mobile phone data. Transactions in GIS, 16(3):375-396, 2012.

12.Li, Y., Liu, H., Liu, G., Li, L., Moore, P., Hu, B.: A grouping method based on grid density and relationship for crowd evacuation simulation. Physica A: Statistical Mechanics and its Applications, 473:319-336, 2017.

13.Sentinel, Accuware. https://www.sentinelcv.com/. [Online; accessed February 15, 2019].

14.Temperature sensors, Texas Instruments incorporated. http://www.ti.com/ sensors/temperatu re-sensors/overview.html. [Online; accessed February 15, 2019].

15.Intelligent smoke detectors, Kidde-Fenwal Systems. https://kidde-fenwal.com/Public/Syst emDetails/Kidde-Fire-Systems/SmartOne-Intelligent-Smoke-Detectors. [Online; accessed February 15, 2019].

16.Talebi, K., and Smith, J.M.: Stochastic network evacuation models. Computers \& Operations Research, 12(6):559-577, 1985. 